VOL. $16(1977), 131-145$.

\title{
Varieties of a closed category
}

\section{B.J. Day}

In this article closed variety structures generated by a monoidal class of identities are examined. This leads to closed enrichments of various results of Y. Diers [Am. Soc. Sci. Bruxelzes Sér. I 90 (1976), 159-172] on varieties of a category.

\section{Introduction}

The general concept of a variety of a category relative to a fully faithful dense functor $N: A+C$ and a class of $N$-identities is due to Diers [5]. It leads to the generalisation of various results for algebraic theories by Birkhoff and Mal'cev concerning varieties of algebras, by Shafaat concerning classes of algebras defined by implications, and by Hatcher concerning quasi-primitive classes of algebras.

The aim in this article is to introduce the concept of a monoidal class of $N$-identities for a monoidal category $A$ and a fully faithful dense functor from $A$ to a monoidal biclosed category $C$. This leads to the idea of a biclosed $N$-variety and to the operations of taking the monoidal interior and closure of a class of $N$-identities or a class of $N$-implications corresponding, by "duality", to taking the biclosed closure and biclosed interior of an $N$-variety.

Because most of the work consists of monoidal biclosed enrichment of the results of Diers [5] we assume some familiarity with this article. We also assume some familiarity with the basic properties of category theory and closed category theory (as given, for example, in Mac Lane [8] and Eilenberg and Kelly [6]).

Received 28 September 1976. The author gratefully acknowledges the support of a Post-Doctoral Fellowship from the Australian Research Grants Comittee. 
We note that most of the results treated here remain valid if all the categorical algebra is relative to a cartesian closed category $V$ having all small limits and colimits. However we here deal only with the case $V=S$, the cartesian closed category of small sets.

\section{Preliminaries}

A monoidal category $A=(A, I, \otimes, \ldots)$ is as defined in Eilenberg and Kelly ([6], Chapter II). A monoidal category $C$ is said to be biclosed if both the functors $-\otimes C: C \rightarrow C$ and $C \otimes-: C \rightarrow C$ have right adjoints

$$
\begin{aligned}
& C(B \otimes C, D) \cong C(C, B \backslash D), \\
& C(C \otimes B, D) \cong C(C, D / B) .
\end{aligned}
$$

A symmetric monoidal closed category (Eilenberg and Kelly [6], Chapter III) is a particular example of a monoidal biclosed category. To each result in the sequel there will be a corresponding result-with-symmetry which we shall not state explicitly.

An important structure theorem for monoidal biclosed categories is the following:

THEOREM 1.1. Let $\mathrm{A}$ be a promonoidal category and let $N: \mathrm{A}^{\circ \mathrm{P}} \rightarrow \mathrm{C}$ be a dense functor. Then $C$ admits a monoidal biclosed structure such that $N^{\circ p}: A \rightarrow C^{\circ p}$ admits enrichment to a strong promonoidal functor if and only if the following "mean" tensor products and cotensor products exist in $C$ for all $A, A^{\prime} \in A$ and $C, C^{\prime} \in C$,

$$
\begin{aligned}
Q\left(A A^{\prime}\right)= & P\left(A A^{\prime} X\right) \circ N X, \\
& J X \circ N X, \\
& \left(C(N X, C) \times C\left(N X^{\prime}, C^{\prime}\right)\right) \circ Q\left(X X^{\prime}\right), \\
H(A C)= & C(Q(X A), C) \circ N X, \\
K(A C)= & C(Q(A X), C) \circ N X, \\
& \left\{C(N X, C), H\left(X C^{\prime}\right)\right\}, \\
& \left\{C(N X, C), K\left(X C^{\prime}\right)\right\},
\end{aligned}
$$

and the resulting morphisms

$$
C\left(Q\left(A^{\prime} A\right), C\right) \rightarrow C\left(N A^{\prime}, H(A C)\right),
$$




$$
\begin{aligned}
& \text { Varieties of a closed category } \\
& \qquad\left(Q\left(A A^{\prime}\right), C\right) \rightarrow C\left(N A^{\prime}, K(A C)\right)
\end{aligned}
$$

are isomorphisms for all $A, A^{\prime} \in A$ and $C \in C$. The resulting monoidal biclosed structure on $C$ is then uniquely determined to within isomorphism. $\quad / /$

When the hypotheses of Theorem 1.1 are satisfied we say that $C$ is the Kan extension of $A^{\circ p}$ along $N$. For the proof of this theorem we refer to Day [4]. For the concept of a promonoidal category we refer to Day [2], and for the concept of "mean" tensor product "o" and cotensor product " $\{-,-\}$ " we refer to Borceux and Kelly [1].

If $M$ is a full subcategory of a monoidal biclosed category $C$ which is closed under isomorphs in $C$ we say that $M$ is closed under exponentiation in $\mathrm{C}$ if $C \backslash M \in \mathrm{C}$ and $M / C \in \mathrm{C}$ for all $C \in \mathrm{C}$ and $M \in M$. Note that this results in a "biclosed" structure on $M$ which, in general, lacks an identity unless $M$ is reflective in $C$ (in which case $M$ is monoidal biclosed by Day [3]; this is actually a special instance of Theorem 1.1).

We recall the following:

LEMMA 1.2. Suppose $\mathrm{A} \subset \mathrm{B} \subset \mathrm{C}$ and $\mathrm{A} \subset \mathrm{C}$ is dense. Then $\mathrm{B} \subset \mathrm{C}$ is dense.

Proof. Suppose $\alpha_{B}: C(B C) \rightarrow C(B D)$ is natural in $B \in \mathrm{B}$. Then its restriction $\alpha_{A}: C(A C) \rightarrow C(A D)$ is natural in $A \in A$. By density of $A$, $\alpha_{A}=C(A, f)$ for a unique morphism $f \in C(C D)$. Thus $\alpha_{B}=C(B, f)$ for a unique morphism $f \in C(C D)$ if the diagram

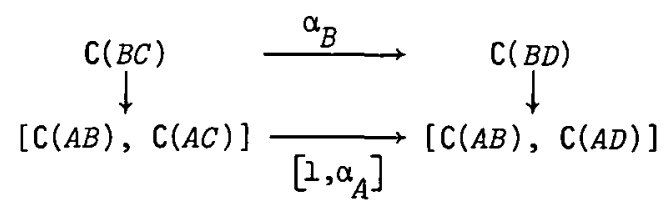

commutes for all $A \in A$ and $B \in B$, which it does by naturality of $\alpha_{B}$ in $B \in \mathrm{B} . \quad / /$

Note that this lemma can not be established over an arbitrary base category $V$ unless $V: V \rightarrow S$ is faithful. 
Given that a monoidal biclosed category $C$ is the Kan extension of $A^{\circ p}$ along $N: A^{O P} \rightarrow C$, for some promonoidal structure on $A$, we can form

$$
\tilde{\mathrm{A}}=\left\{N A_{1} \otimes \ldots \otimes N A_{n} ; A_{1}, \ldots, A_{n} \in \mathrm{A}\right\} \cup\{I\}
$$

and deduce, from Theorem 1.1 and Lemma 1.2 , that $C$ is the Kan extension of $\tilde{A}$ along the dense embedding $\tilde{N}: \tilde{A} \rightarrow C$.

\section{Monoidal classes of $N$-identities and $N$-implications}

We commence by supposing that $N: A \rightarrow C$ is a fully faithful dense functor from a monoidal category $A$ to a monoidal biclosed category $C$, where $C$ has all small limits and $N$ preserves tensor products (including the "empty" tensor product $I$ ). Thus $C$ is the Kan extension of A along $N$.

A family $F$ of $N$-identities is said to be monoidal if $\left(\omega_{1}=\omega_{2}\right) \in F$ implies $\left(\omega_{1} \otimes A=\omega_{2} \otimes A\right) \in F$ and $\left(A \otimes \omega_{1}=A \otimes \omega_{2}\right) \in F$ for all $A \in A$. Similarly a family $F$ of $N$-implications is called monoidal if whenever

$$
\wedge\left(\omega_{1}^{i}=\omega_{2}^{i}\right) \Rightarrow \mu_{1}=\mu_{2}
$$

is in $F$ then both

$$
\wedge\left(\omega_{1}^{i} \otimes A=\omega_{2}^{i} \otimes A\right) \Rightarrow \mu_{1} \otimes A=\mu_{2} \otimes A
$$

and

$$
\wedge\left(A \otimes \omega_{1}^{i}=A \otimes \omega_{2}^{i}\right) \Rightarrow A \otimes \mu_{1}=A \otimes \mu_{2}
$$

are in $F$ for all $A \in A$.

PROPOSITION 2.1. If a full subcategory $M$ of $C$ is defined by $a$ monoidal family of $N$-identities or by a monoidal family of $N$-implications then $M$ is closed under products, subobjects, and exponentiation in $C$.

Proof. It is straightforward to see that $M$ is closed under products and subobjects in C (see Diers [5], Proposition 1.1). Moreover, because the defining family is monoidal, $M$ is closed in $C$ under exponentiation 
of the form $N A W$ and $M / N A$ for all $A \in \mathrm{A}$. Thus, because $\mathrm{C}$ is the Kan extension of $A, M$ is closed under all exponentiation in $C$. //

Given a family $F$ of $N$-identities we can associate with $F$ its monoidal interior $F^{O}$ and its monoidal closure $\bar{F}$ : $F^{0}=\left\{\left(\omega_{1}=\omega_{2}\right) ; \omega_{1} \otimes A=\omega_{2} \otimes A \in F \quad\right.$ and $A \otimes \omega_{1}=A \otimes \omega_{2} \in F$ for all $A \in A\}$, $F=\left\{\left(\omega_{1}^{\prime}=\omega_{2}^{\prime}\right) ; \omega_{1}^{\prime}=A^{\prime} \otimes \omega_{1} \otimes A\right.$ and $\omega_{2}^{\prime}=A^{\prime} \otimes \omega_{2} \otimes A$ where $\left.\left(\omega_{1}=\omega_{2}\right) \in F\right\}$.

Similarly one can associate with any family of $\mathrm{N}$-implications its monoidal interior and monoidal closure.

An $N$-variety of $\mathrm{C}$ is said to be a biclosed $N$-variety if it is closed under exponentiation in $C$. Note that if $F$ defines the $N$-variety $M$ then $F^{0}$ defines a biclosed $N$-variety $\bar{M}$ and $\bar{F}$ defines a biclosed $N$-variety $M^{0}$.

\section{N-implications as $\tilde{N}$-identities}

As in Diers ( $[5], \S 2)$, a multicokernel is a colimit of a diagram $\left(\left(f_{i}, g_{i}\right): A_{i} \rightarrow A_{0}\right)_{i \in I}$ formed by pairs of morphisms having a common domain. If $|I|<\alpha$ then this colimit is called an $\alpha$-multicokernel.

Suppose that $C$ has multicokernels and regular factorisations. Let $\tilde{A}_{\alpha}$ be the full subcategory of $C$ comprising the finite tensor products of $\alpha$-multicokernels in $\mathrm{C}$ and let $\tilde{N}_{\alpha}$ denote the inclusion functor $\tilde{\mathrm{A}}_{\alpha} \subset \mathrm{C}$, which is dense by Lemma 1.2 .

PROPOSITION 3.1. If $\alpha$ is an infinite cardinal number then the full subcategories of $\mathrm{C}$ defined by monoidal classes of $N$-implications of length Zess than $\alpha$ are exactly the full subcategories of $\mathrm{C}$ defined by monoidal classes of $\tilde{N}_{\alpha}$-identities.

Proof. The proof is similar to that of Diers ([5], Proposition 2.0).

(a) Let $F$ be a monoidal family of $N$-implications. Form the 
monoidal family $\bar{G}$ of $\tilde{N}_{\alpha}$-identities, where $G$ comprises the $\tilde{N}_{\alpha}$-identities $\left(k \cdot N \mu_{1}=k \cdot N \mu_{2}\right)$ as $k$ runs through the multicokernels of the "codomains" of the implications in $F$. If $X$ is an F-model then $(H \backslash K) / K$ is an $F$-model for all $\alpha$-multicokernels $H$ and $K$ because $F$ is monoidal (Proposition 2.1). Thus $(B \backslash X) / K$ is a G-model, whence $X$ is a. $\bar{G}$-model. Conversely, if $X$ is a $\bar{G}$-model, then $X$ is a G-model, so $X$ is an F-model.

(b) Let $G$ be a monoidal family of $\tilde{N}_{\alpha}$-identities. Form a class $F$ of $N$-implications as follows: for each $\left(\nu_{1}=\nu_{2}\right)$ in $G$ the domain $K_{1} \otimes \ldots \otimes K_{n}$ is a finite tensor product of $\alpha$-multicokernels, so is an $\alpha$-multicokernel. Suppose $K_{j}(1 \leq j \leq n)$ is the multicokernel of $\left(N \omega_{1}^{i} j, N \omega_{2}^{i} j\right)$ and let $k$ be the coequaliser $\left(v_{1}, v_{2}\right)$ in $c$.

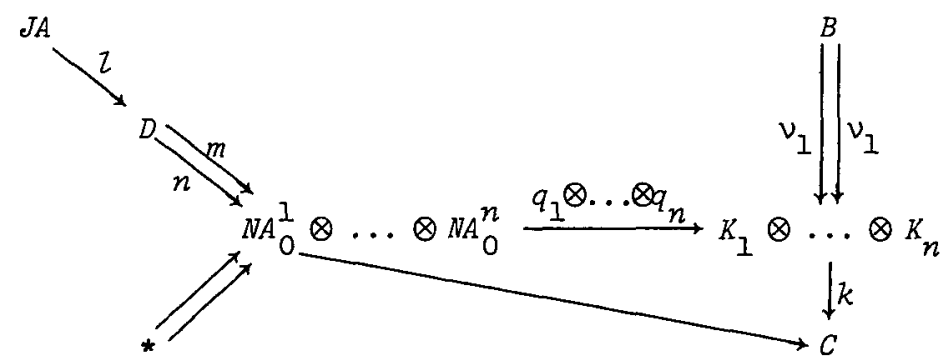

Then $k\left(q_{1} \otimes \ldots \otimes q_{n}\right)$ is a regular epimorphism. Let $(m, n)$ be its difference kernel and, for each $l$ in the comma category $(N, D)$, let $N \mu_{1}^{2}=m \tau$ and $N \mu_{2}^{2}=n \tau$. Let $F$ be the family

$$
\wedge\left\{1 \otimes \omega_{1}^{i} j \otimes I=1 \otimes \omega_{2}^{i} j \otimes 1\right\} \Rightarrow \mu_{1}^{2}=\mu_{2}^{2}
$$

of $\alpha$-implications, where $l \in(N, D)$, and let $\bar{F}$ be its monoidal closure. If $X$ is an $\bar{F}$-model, then $X$ is an F-model, so each $y: K_{1} \otimes \ldots \otimes K_{n} \rightarrow X$ has the property that $y\left(q_{1} \otimes \ldots \otimes q_{n}\right)$ coequalises $(m l, n l)$ for all $l(N, D)$. Thus, because $N$ is dense, $y\left(q_{I} \otimes \ldots \otimes q_{n}\right)$ coequalises $(m, n)$, so $y$ factors through $k$ and $X$ is a G-model. Conversely, if $X$ is a $G$-model, then $(H \backslash X) / K$ is a 
G-model for all $\alpha$-multicokernels $H$ and $K$ because $G$ is monoidal (Proposition 2.1). Then every $x: N A_{0}^{l} \otimes \ldots \otimes N A_{0}^{n} \rightarrow(H \backslash X) / K$ which factors as $y\left(q_{1} \otimes \ldots \otimes q_{n}\right)$ factors through $k$, as required to coequalise $(m l, n \tau)$ for all $l \in(N, D)$. Thus $(H \backslash X) / K$ is an F-model for all $\alpha$-multicokernels $H$ and $K$ so $X$ is an $\bar{F}$-model. //

\section{Proper $N$-reflective subcategories}

Let $\phi: M \rightarrow C$ be a full $N$-reflective embedding into $C$ with $N$-adjoint $\psi: \mathrm{A} \rightarrow \mathrm{M}$ and unit $\mathrm{n}: N \rightarrow \phi \psi$; this concept is due to Ulmer [9]. Then, as in Diers ([5], $\$ 3)$, the $N$-reflection is called proper (equals véritable) if $X \in M$ whenever ${ }^{\prime} C(\eta, X)$ is an isomorphism.

We recall from Diers $([5], \S 3)$ that an $N$-reflective subcategory $M$ of $\mathrm{C}$ is proper if $\mathrm{M}$ is closed under $N$-absolute colimits. Also, if $\mathrm{C}$ has regular factorisations, then a proper $N$-reflective subcategory of $C$ is an $N$-variety if and only if it is closed under subobjects.

THEOREM 4.1. Let $\phi: M \rightarrow C$ be a proper $N$-reflective subcategory of a monoidal biclosed category $C$ with $\psi \vec{N} \phi: M \rightarrow C$ and unit $\eta: N+\phi \psi$. Then the following conditions are equivalent:

(1) $C \backslash \phi M$ and $\phi M / C$ are in $C$ for alz $C \in C$ and $M \in M$;

(2) $N A \backslash \phi M$ and $\phi M / N A$ are in $C$ for all $A \in \mathrm{A}$ and $M \in M$;

(3) $N A \backslash \phi M \cong \phi \psi A \backslash \phi M$ and $\phi M / N A \cong \phi M / \phi \psi A$ for all $A \in A$ and $M \in \mathrm{M}$.

Proof. $C \backslash \phi M \in C$ for all $C \in C$ and $M \in M$ if and only if

$$
\mathrm{C}\left(\eta_{A}, C \backslash \phi M\right): \mathrm{C}(\phi \psi A, C \backslash \phi M) \cong \mathrm{C}(N A, C \backslash \phi M)
$$

for all $A \in \mathrm{A}, C \in \mathrm{C}, M \in M$, if and only if

$$
\mathrm{C}\left(C, \phi M / \mathrm{n}_{A}\right): \mathrm{C}(C, \phi M / \phi \psi A) \cong \mathrm{C}(C, \phi M / N A)
$$

for all $A \in A, C \in C, M \in M$, if and only if $\phi M / \phi \psi A \cong \phi M / N A$ for all $A \in A$ and $M \in M$. Also $N A \backslash \phi M \in C$ for all $A \in A$ and $M \in M$ if and only if

$$
\mathrm{C}\left(\eta_{A^{\prime}}, N A \backslash \phi M\right): \mathrm{C}\left(\phi \psi A^{\prime}, N A \backslash \phi M\right) \cong \mathrm{C}\left(N A^{\prime}, N A \backslash \phi M\right)
$$

for all $A, A^{\prime} \in \mathrm{A}$ and $M \in \mathrm{M}$, if and only if 


$$
\mathrm{C}\left(N A, \phi M / \eta_{A^{\prime}}\right): \mathrm{C}\left(N A, \phi M / \phi \psi A^{\prime}\right) \cong \mathrm{C}\left(N A, \phi M / N A^{\prime}\right)
$$

for all $A, A^{\prime} \in A$ and $M \in M$, if and only if $\phi M / \phi \psi A \cong \phi M / N A$ for all $A \in A$ and $M \in M$, since $N$ is dense.

COROLLARY 4.2. Suppose the conditions of Theorem 4.1 are satisfied. If $A$ is monoidal and $N: A \rightarrow C$ is monoidal, then $\theta=\phi \psi: A \rightarrow C$ is monoidal and $\eta: N \rightarrow \phi \psi$ is a monoidal natural transformation.

Proof. Define $\tilde{\theta}: \phi \psi A \otimes \phi \psi A^{\prime} \rightarrow \phi \psi\left(A \otimes A^{\prime}\right)$ to be the image of $\tilde{N}: N A \otimes N A^{\prime} \rightarrow N\left(A \otimes A^{\prime}\right)$ under the transformation

$$
\begin{aligned}
C\left(N A \otimes N A^{\prime}, N\left(A \otimes A^{\prime}\right)\right) \frac{\bar{C}\left(1, n_{A \otimes A^{\prime}}\right)}{} & C\left(N A \otimes N A^{\prime}, \phi \psi\left(A \otimes A^{\prime}\right)\right) \\
& \cong C\left(N A^{\prime}, N A \phi \psi\left(A \otimes A^{\prime}\right)\right) \\
& \cong C\left(N A^{\prime}, \phi \psi A \phi \psi\left(A \otimes A^{\prime}\right)\right) \\
& \cong C\left(\phi \psi A, \phi \psi\left(A \otimes A^{\prime}\right) / N A^{\prime}\right) \\
& \cong C\left(\phi \psi A, \phi \psi\left(A \otimes A^{\prime}\right) / \phi \psi A^{\prime}\right) \\
& \cong C\left(\phi \psi A \otimes \phi \psi A^{\prime}, \phi \psi\left(A \otimes A^{\prime}\right)\right)
\end{aligned}
$$

where the isomorphism is easily seen to be $c\left(\eta_{A} \otimes \eta_{A}, 1\right)$. Similarly define $\theta^{0}: I \rightarrow \phi \psi I$ to be

$$
I \stackrel{\tilde{N}}{\longrightarrow} N I \stackrel{\eta_{I}}{\longrightarrow} \phi \psi I
$$

The axioms of a monoidal functor $\theta=\left(\theta, \tilde{\theta}, \theta^{0}\right)$ are straightforward. //

If $C$ has regular factorisations and each object of $C$ has only a set of strong quotients then each $N$-variety $M$ of $C$ is reflective in $C$ by the general adjoint functor theorem; each biclosed $N$-variety is then a monoidal biclosed category (by the biclosed version of Day [3], Theorem 1.2). In these circumstances, if $N: A \rightarrow C$ preserves tensor products, $M$ is the Kan extension of $A$ along $\psi: A \rightarrow M$, noting that $\psi$ is dense by virtue of the isomorphism $C(N A, \phi M) \cong M(\psi A, M)$ and the density of $N$.

PROPOSITION 4.3. If $C$ has regular factorisations, then a proper biclosed N-reflective subcategory of $\mathrm{C}$ is a biclosed N-variety if and only if it is closed under subobjects.

Proof. The condition is necessary by Proposition 2.1. Conversely, suppose $M$ is a proper biclosed $N$-reflective subcategory of $C$ which is closed under subobjects. Then we construct 


$$
F=\left\{\omega_{1}=\omega_{2} ;\left(\omega_{1}, \omega_{2}\right): A_{1} \rightarrow A_{0}, \eta_{A_{0}} \cdot N \omega_{1}=n_{A_{0}} \cdot N \omega_{2}\right\} .
$$

This $F$ defines $M$ as an $N$-variety by Diers ([5], Proposition 3.2). Moreover $F$ is monoidal because, for example,

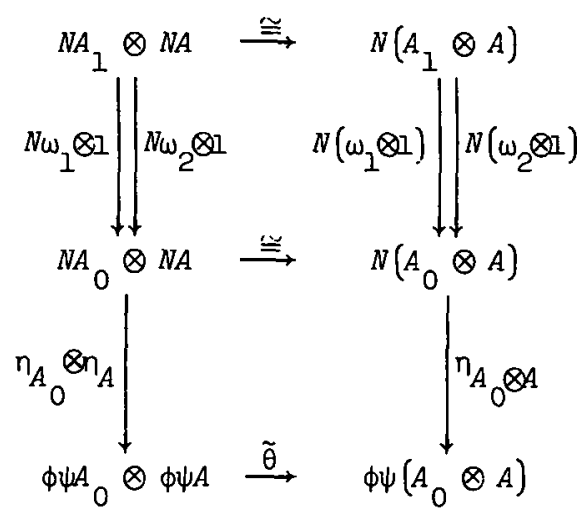

commutes (see Corollary 4.2).

\section{Monoidal density presentations}

We recall (Day [4], §8) that a density presentation of $N: A \rightarrow C$ comprises a coefficient functor $K: K^{O P} \times|C| \rightarrow S$, an index functor $J: \mathrm{K} \rightarrow \mathrm{A}$, and a structure transformation $\xi_{C}: K(k, C) \circ N J k \cong C$ for each $C \in|C|$ such that the induced transformation

$$
\int^{k} K(k, C) \times \mathrm{A}(A, J k) \rightarrow \mathrm{C}(N A, C)
$$

is an isomorphism for all $A \in A$ and $C \in C$. Here $|C|$ denotes the discrete category on $C$.

If $C$ is the Kan extension of a monoidal structure on $A$ along $N$, then we call a density presentation of $N$ a monoidal presentation if the induced transformation

$\int^{k l} K(k, C) \times K(Z, D) \times A(A, J k \otimes J Z)$

$$
\rightarrow C(N A,(K(k, C) \times K(\tau, D)) \circ N(J k \otimes J l))
$$

is an isomorphism for all $A \in A$ and $C, D \in C$. Thus, if $N$ is a full embedding, a presentation of $N$ is monoidal if and only if the expression 


$$
C \otimes D=(K(k, C) \times K(Z, D)) \circ N(J k \otimes J Z)
$$

derived from the given density presentation of $N$ is an $N$-absolute colimit. Thus, by Diers ([5], Theorem 4.0), we have

THEOREM 5.1. For a monoidal density presentation of $N: A \rightarrow C$ every biclosed $N$-variety is monoidai and closed under tensor products in C. $/ 1$

PROPOSITION 5.2. If $(K, J, \xi)$ is a monoidal presentation of $N: A \rightarrow C$ then, for each functor $G: A^{O P} \rightarrow S$, there exists an isomorphism

$$
\int_{A}[C(N A, C \otimes D), G A] \cong \int_{k Z}[K(k, C) \times K(\tau, D), G(J k \otimes J Z)]
$$

for a $C, D \in \mathrm{C}$.

Proof. This follows from the representation theorem.

$$
/ /
$$

\section{Examples}

EXAMPLE 6.1. Let $\alpha$ be a regular cardinal number. We say that a cartesian closed category $V$ is $\alpha$-cartesian if it has $\alpha$-products and the property that

$$
\int^{\pi A_{\lambda}} \pi S_{\lambda}\left(A_{\lambda}, A_{\lambda}\right) \rightarrow \pi \int^{A_{\lambda}} S_{\lambda}\left(A_{\lambda}, A_{\lambda}\right)
$$

is an isomorphism for any family

$$
\left\{S_{\lambda}: A^{o p} \times A \rightarrow V ; \lambda \in \Lambda\right\}
$$

of V-functors with $|\Lambda|<\alpha$ for which each $\int^{A_{\lambda}} S_{\lambda}\left(A_{\lambda}, A_{\lambda}\right)$ exists.

Any cartesian closed category is $|N|$-cartesian closed, and probably one should consider the corresponding notion of an $\alpha$-closed category; but we do not need it here since we are dealing only with binary monoidal structures.

PROPOSITION 6.1.1. Let $\alpha$ be a regular cardinal number and $V$ be an a-cartesian category. Let $\mathrm{M}: \mathrm{A} \rightarrow \mathrm{B}$ be a $\mathrm{V}$-functor between 
V-categories with $V$ - $\alpha$-products. Then $\operatorname{lan}_{M} G: B \rightarrow V$, whenever it exists, preserves a-products whenever $G: A \rightarrow V$ does:

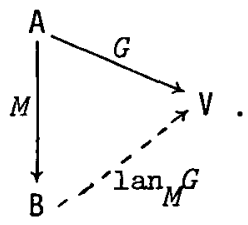

Proof. Let ${ }_{\Lambda}^{\pi B} \lambda$ be a $V$ - $\alpha$-product in $B$. Then

$$
\begin{aligned}
\operatorname{lan}_{M} G\left(\pi B_{\lambda}\right) & =\int^{A} \mathrm{~B}\left(M A, \pi B_{\lambda}\right) \times G A \\
& \cong \int^{A} \pi \mathrm{B}\left(M A, B_{\lambda}\right) \times G A \\
& \cong \int^{A} \pi\left(\int^{X} \mathrm{~B}\left(M X_{\lambda}, B_{\lambda}\right) \times \mathrm{A}\left(A, X_{\lambda}\right)\right) \times G A
\end{aligned}
$$

by the representation theorem

$\cong \int^{\pi A_{\lambda}} \pi \mathrm{B}\left(M X_{\lambda}, B_{\lambda}\right) \times \int^{A} \pi \mathrm{A}\left(A, X_{\lambda}\right) \times G A$

because $V$ is $\alpha$-cartesian

$\cong \int^{\pi A_{\lambda}} \pi \mathrm{B}\left(M X_{\lambda}, B_{\lambda}\right) \times \int^{A} \mathrm{~A}\left(A, \pi X_{\lambda}\right) \times G A$

$\cong \int^{\pi A_{\lambda}} \pi \mathrm{B}\left(M X_{\lambda}, B_{\lambda}\right) \times G\left(\pi X_{\lambda}\right)$ by the representation theorem

$\cong \int^{\pi A_{\lambda}} \pi \mathrm{B}\left(M X_{\lambda}, B_{\lambda}\right) \times \pi G X_{\lambda}$ because $G$ preserves $V_{-\alpha \text {-products }}$

$\cong \int^{\pi A} \pi\left(B\left(M X_{\lambda}, B_{\lambda}\right) \times G X_{\lambda}\right)$

$\cong \pi \int^{X} \mathrm{~B}\left(M X_{\lambda}, B_{\lambda}\right) \times B X_{\lambda}$ because $V$ is $\alpha$-cartesian

$=\pi \operatorname{lan}_{M} G\left(B_{\lambda}\right)$. /

Now suppose that. $A$ is a small $V$-monoidal category with

$V$ - $\alpha$-coproducts where $V$ is assumed to be $\alpha$-cartesian closed with all 
small limits and colimits. Let $\left[A^{\circ p}, V\right]_{\alpha} \subset\left[A^{o p}, V\right]$ be the full subcategory of $V$-functors which preserve $\alpha$-products (relative to $V$ ). Let $N: \mathrm{A} \rightarrow\left[\mathrm{A}^{\mathrm{OP}}, V\right]_{\alpha}$ be the Yoneda embedding. Consider the convolution structure on $\left[A^{\circ p}, V\right]$ given by

$$
F \otimes G=\int^{A A^{\prime}} F A \times G A^{\prime} \times A\left(-, A \otimes A^{\prime}\right)
$$

(see Day, [2]).

PROPOSITION 6.1.2. If $F$ and $G$ preserve $V$ - $\alpha$-products, so does $F \otimes G$.

Proof. $F \otimes G: A^{\circ p} \rightarrow V$ is the left Kan extension of $E \times G: \mathrm{A}^{\mathrm{OP}} \times \mathrm{A}^{\mathrm{OP}} \rightarrow \mathrm{V}$ along $\otimes: A \times A \rightarrow A$. But $F \times G$ preserves $V$ - $\alpha$-products, since

$$
\begin{aligned}
F \times G\left(\pi\left(A_{\lambda}, A_{\lambda}^{\prime}\right)\right) & =F \times G\left(\left(\pi A_{\lambda}, \pi A_{\lambda}^{\prime}\right)\right) \\
& =F\left(\dot{\pi}_{\lambda}\right) \times G\left(\pi A_{\lambda}^{\prime}\right) \\
& \cong \pi F A_{\lambda} \times \pi G A_{\lambda} \\
& \cong \pi\left(F A_{\lambda} \times G A_{\lambda}\right) \\
& \cong \pi F \times G\left(A_{\lambda}, A_{\lambda}^{\prime}\right) .
\end{aligned}
$$

Thus the result follows from Proposition 6.1.1. //

This implies that the usual Yoneda presentation of $A \rightarrow\left[A^{\circ p}, V\right]_{\alpha}$ is a V-monoidal-density presentation. In particular, by Theorem 5.1 and Diers ([5], Proposition 5.0.0), we have

PROPOSITION 6.1.3. A full subcategory of $\left[\mathrm{A}^{\mathrm{Op}}, \mathrm{S}\right]_{\alpha}$ is a biclosed $N$-variety if and only if it is closed under products, subobjects, exponentiation, $\alpha$-filtered colimits, regular quotients, and tensor products. $/ /$

REMARK 6.1.4. In any monoidal biclosed $N$-variety such as that defined by Proposition 6.1.3 the $n$th tensor-power functor is described by: 


$$
\begin{aligned}
& F^{(n)}=F \otimes \ldots \otimes F=\int^{A_{1} \ldots A_{n}}{ }_{F A_{1}} \times \ldots \times F A_{n} \times \mathrm{A}\left(-, A_{1} \otimes \ldots \otimes A_{n}\right) \\
& \cong \int^{A_{1} \cdots A_{n}} F\left(A_{1}+\ldots+A_{n}\right) \times \mathrm{A}\left(-, A_{1} \otimes \ldots \otimes A_{n}\right) \\
& \text { because } F \text { preserves finite products } \\
& \cong \int^{B A_{1} \ldots A_{n}} F B \times \mathrm{A}\left(A_{1}+\ldots+A_{n}, B\right) \\
& \times A\left(-, A_{1} \otimes \ldots \otimes A_{n}\right) \text { by the representation theorem } \\
& \cong \int^{B} F B \times A\left(-, B^{(n)}\right) \text { by the representation theorem. }
\end{aligned}
$$

This holds for $n \geq 1$, and similarly

$$
F^{(0)} \cong \int^{B} F B \times \mathrm{A}\left(-, B^{(0)}\right) \cong \mathrm{A}(-, I)
$$

In particular, if $A \otimes A^{\prime}$ is taken to be $A+A^{\prime}$ in $\mathrm{A}$, then (the $\otimes$-algebra structure on) each object of $A$ induces a $\otimes$-algebra structure on each functor $F \in\left[\mathrm{A}^{\mathrm{Op}}, \mathrm{S}\right]_{\alpha}$, where $\mu: F \otimes F \rightarrow F$ is given by

$$
F \otimes F(A) \cong \int^{B} F B \times A(A, B \otimes B)+F A,
$$

corresponding to $F \mu: F B \rightarrow F(B \otimes B)$ by the representation theorem, and similarly for $\eta: A(-, I) \rightarrow F$. This remark still holds if $S$ is replaced by a suitable cartesian closed category $V$.

EXAMPLE 6.2. Let $T=(T, \eta, \mu)$ be a monoidal monad on a sufficiently complete and cocomplete monoidal biclosed category $B$. Suppose that $T: B \rightarrow B$ preserves coequalisers of reflective pairs of morphisms. Then $T$ is regular since kernel pairs are reflective, and $B^{T}$ is cocomplete by Linton [7].

Let $A=B_{T}$ be the Kleisli category of $T$, let $C=B^{T}$, and let $N: \mathrm{A} \rightarrow \mathrm{C}$ be the dense comparison functor. Then the "standard" density presentation of $N$ (see Day [4], §8) is generated by the usual presentation of each algebra $(C, \xi) \in C$ as a $U^{\top}$-split coequaliser: 


$$
T^{2} C \underset{T \xi}{\stackrel{\mu}{\longrightarrow}} T C \stackrel{\xi}{\longrightarrow} C
$$

where $U^{\top}: C \rightarrow B$ is the underlying-object functor.

The presentation of $C \otimes D$ derived from this presentation of $C$ and $D$ is given as the coequaliser of the reflective pair

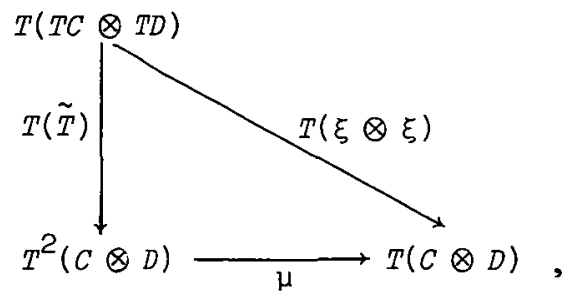

where the reflection is $T(n \otimes \eta)$; see Day ([4], Proposition 4.4).

Now suppose that each of the representable functors $B(B,-): B \rightarrow S$ preserves coequalisers of reflective pairs. Then coequalisers of reflective pairs are $N$-absolute colimits and, moreover, the above presentation of $C \otimes D$ is $N$-absolute; thus the "standard" presentation of $N$ is now monoidal.

Thus from Theorem 5.1 and Diers ([5], Theorem 4.0), we have

THEOREM 6.2.1. A class of T-algebras is a biclosed N-variety of T-algebras if and only if it is closed under products, subobjects, exponentiation, regular quotients, and tensor products. //

\section{References}

[1] Francis Borceux and G.M. Kelly, "A notion of limit for enriched categories", Bulz. Austral. Math. Soc. 12 (1975), 49-72.

[2] Brian Day, "On closed categories of functors", Reports of the Midwest Category Seminar IV, 1-38 (Lecture Notes in Mathematics, 137. Springer-Verlag, Berlin, Heidelberg, New York, 1970).

[3] Brian Day, "A reflection theorem for closed categories", J. Pure Appl. AZgebra 2 (1972), 1-11. 
[4] Brian Day, "On closed categories of functors II", Category seminar, 20-54 (Proc. Sydney Category Theory Seminar 1972/1973. Lecture Notes in Mathematics, 420. Springer-Verlag, Berlin, Heidelberg, New York, 1974).

[5] Y. Diers, "Variétés d'une catégorie", Ann. Soc. Sci. Bruxelzes Ser. I 90 (1976), 159-172.

[6] Samuel Eilenberg and G. Max Kelly, "Closed categories", Proc. Conf. Categorical Algebra, La Jolla, California, 1965, $421-562$ (Springer-Verlag, Berlin, Heidelberg, New York, 1966).

[7] F.E.J. Linton, "Coequalizers in categories of algebras", Seminar on triples and categorical homology theory, 75-90 (Lecture Notes in Mathematics, 80. Springer-Verlag, Berlin, Heidelberg, New York, 1969).

[8] S. Mac Lane, Categories for the working mathematician (Graduate Texts in Mathematics, 5. Springer-Verlag, Berlin, Heidelberg, New York, 1971).

[9] Friedrich Ulmer, "Properties of dense and relative adjoint functors", J. Algebra 8 (1968), 77-95.

Department of Pure Mathematics,

University of Sydney,

Sydney,

New South Wales. 